\title{
A RELAÇÃO DA ENFERMAGEM COM OS ERROS DE MEDICAÇÃO: UMA REVISÃO INTEGRATIVA
}

\author{
Elaine Cristina Novatzki Forte ${ }^{1}$, Francele Luz Machado ${ }^{2}$, Denise Elvira Pires de Pires ${ }^{3}$
}

\begin{abstract}
RESUMO: Revisão integrativa com o objetivo de identificar na literatura a relação dos erros de medicação com a equipe de enfermagem. A pesquisa foi realizada por duas colaboradoras independentes, em três bases de dados, no período 2011 a 2015, com 32 estudos. Os resultados foram divididos em duas macrocategorias: características dos estudos e as relações diretas e indiretas da enfermagem com os erros de medicação. As relações diretas se referem às questões do âmbito profissional, como a falta de conhecimento e de experiência, falhas na comunicação, estresse e distração dos profissionais. As relações indiretas são compostas por questões de prescrição e dispensação, as condições físicas do paciente e erros na produção dos medicamentos. Conclui-se que, além de a enfermagem atuar na administração de medicamentos com vistas à assistência segura ao paciente, pode atuar como barreira dos erros provenientes de outras fases do processo de medicação.

DESCRITORES: Enfermagem; Erros de Medicação; Segurança do Paciente.
\end{abstract}

\section{NURSING'S RELATIONSHIP WITH MEDICATION ERRORS: AN INTEGRATIVE REVIEW}

\begin{abstract}
This integrative review aimed to identify in the literature the relationship between medication errors and the nursing team. The study was undertaken by two independent collaborators, in three databases, in the period 2011 - 2015, with 32 studies. The results were divided in two macrocategories: characteristics of the studies, and the direct and indirect relationships between nursing and the medication errors. The direct relationships refer to issues of the professional ambit, such as lack of knowledge and of experience, failures in communication, stress and the distraction of the professionals. The indirect relationships are made up of issues related to prescribing and dispensing, the patient's physical conditions, and errors in the production of the medications. It is concluded that, besides the nursing staff working in administering medications with a view to safe care for the patient, they can act as a barrier to errors arising from other phases of the medication process.
\end{abstract}

DESCRIPTORS: Nursing; Medication Errors; Patient Safety.

\section{LA RELACIÓN DE LA ENFERMERÍA CON LOS ERRORES DE MEDICACIÓN: UNA REVISIÓN INTEGRATIVA}

RESUMEN: Revisión integrativa cuyo objetivo fue identificar, en la literatura, la relación de los errores de medicación con el equipo de enfermería. Se realizó la investigación por dos colaboradoras independientes, en tres bases de datos, en el periodo de 2011 a 2015, con 32 estudios. Los resultados fueron organizados en dos macrocategorías: características de los estudios y las relaciones directas y indirectas de la enfermería con los errores de medicación. Las relaciones directas se refieren a las cuestiones del ámbito profesional, como la falta de conocimiento y de experiencia, faltas en la comunicación, estrés y distracción de los profesionales. Las relaciones indirectas se constituyen por cuestiones de prescripción y dispensación, las condiciones físicas del paciente y errores en la producción de los medicamentos. Se concluye que, además de la enfermería actuar en la administración de medicamentos para una asistencia segura al paciente, puede actuar como barrera de los errores provenientes de otras fases del proceso de medicación. DESCRIPTORES: Enfermería; Errores de Medicación; Seguridad del Paciente.

${ }^{1}$ Enfermeira. Doutoranda em Enfermagem. Universidade Federal de Santa Catarina. Florianópolis, SC, Brasil.

${ }^{2}$ Enfermeira. Doutora em Enfermagem. Docente de Enfermagem da Universidade Federal de Santa Catarina. Florianópolis, SC, Brasil.

${ }^{3}$ Dicente de Enfermagem. Universidade Federal de Santa Catarina. Florianópolis, SC, Brasil.

Autor Correspondente:

Elaine Cristina Novatzki Forte

Universidade Federal de Santa Catarina

R. Jurerê Tradicional, 1097 - 88053-750 - Florianópolis, SC, Brasil

Email: elainecnforte@gmail.com
Recebido: 01/10/2015

Finalizado: $27 / 07 / 2016$ 


\section{INTRODUÇÃO}

Os erros de medicação estão entre os eventos adversos mais frequentes na assistência à saúde, e que, em sua maioria, poderiam ter sido evitados em uma das fases do processo de medicação (prescrição, dispensação e administração). A prevenção desses erros só é possível diante do levantamento dos fatores envolvidos nesse processo, a fim de criar e implementar barreiras e, com isso, diminuir os riscos à saúde dos pacientes ${ }^{(1,2)}$.

Entende-se por erro de medicação qualquer evento suscetível de prevenção, que pode culminar em uso inadequado de medicamentos, e esses erros tem relação com diferentes fatores ${ }^{(3)}$. Dentre esses fatores, a literatura destaca, especialmente ${ }^{(4-7)}$ : omissão, erro de dose e horário, erro de técnica de administração e troca de vias. O mais relevante é o desfecho desse tipo de erro, que pode resultar em aumento do tempo de internação, agravar o quadro de saúde e gerar incapacidades ou o óbito de pacientes ${ }^{(1,2)}$.

O processo de medicação percorre um longo caminho até a administração, propriamente dita, no paciente. Inicia na produção, segue com a prescrição e dispensação, e por fim, a administração. Essa última compreende todo o processo de checagem, diluição, preparação e administração no paciente. A enfermagem é a profissão de saúde responsável pela parte final desse processo, a administração de medicamentos e, portanto, a sua atuação é crucial para evitar erros dessa natureza.

Com base nesse complexo sistema de medicação, esta revisão tem por intuito identificar na literatura atual a relação dos erros de medicação com a equipe de enfermagem.

\section{METODOLOGIA}

Pesquisa documental realizada por duas colaboradoras independentes, no período de 01 a 05 de julho de 2015, utilizando os pressupostos da revisão integrativa de literatura(8,9), orientado pela questão: qual produção científica está sendo construída sobre os erros de medicação relacionados com a equipe de enfermagem? Para tanto, foi elaborado um protocolo de revisão integrativa, como requerem estudos dessa natureza ${ }^{(8,9)}$.

A pesquisa foi realizada nas seguintes bases de dados: Banco de Teses da Coordenação de Aperfeiçoamento de Pessoal de Nível Superior (CAPES); Biblioteca Virtual em Saúde (BVS); Scientific Electronic Library Online - SciELO; e no PubMed (US National Library of Medicine National Institutes of Health) do National Center for Biotechnology Information (NCBI). A chave com os descritores utilizados foi: Enfermagem OR Equipe de enfermagem AND Erros de medicação.

Os estudos incluídos foram constituídos de artigos científicos, teses e dissertações, que continham os descritores no resumo e/ou no título, publicados em inglês e português, no período de 2011 a 2015, na forma completa pelo acesso via Universidade Federal de Santa Catarina (UFSC). Foram excluídas as cartas, resenhas, revisões sistematizadas, livros, capítulos de livros, documentos governamentais, boletins informativos e estudos duplicados (que aparecem em mais de uma base de dados) (Figura 1).

Após a seleção criteriosa, os estudos foram organizados em um software para análise de dados qualitativos, o Atlas.ti. 7.0 e analisados sob os preceitos da análise temática de conteúdo de Bardin ${ }^{(10)}$, seguindo os passos de pré-análise, exploração do material e tratamento e interpretação dos resultados. A partir da leitura exaustiva de cada estudo, deu-se a codificação das informações a fim de obter o núcleo de sentido, e assim destacar as unidades de registro (quotations, na linguagem do software), atribuindo a essas unidades, códigos identificadores (codes). Ao fim, as categorias (families) foram organizadas conforme o objetivo deste estudo, em duas categorias, sendo: características dos estudos encontrados e a relação da enfermagem com os erros de medicação. A amostra final desta pesquisa foi composta por 32 estudos.

Tendo em vista ser esta uma revisão integrativa de literatura, dispensou-se a avaliação prévia por um Comitê de Ética em Pesquisas com Seres Humanos. 


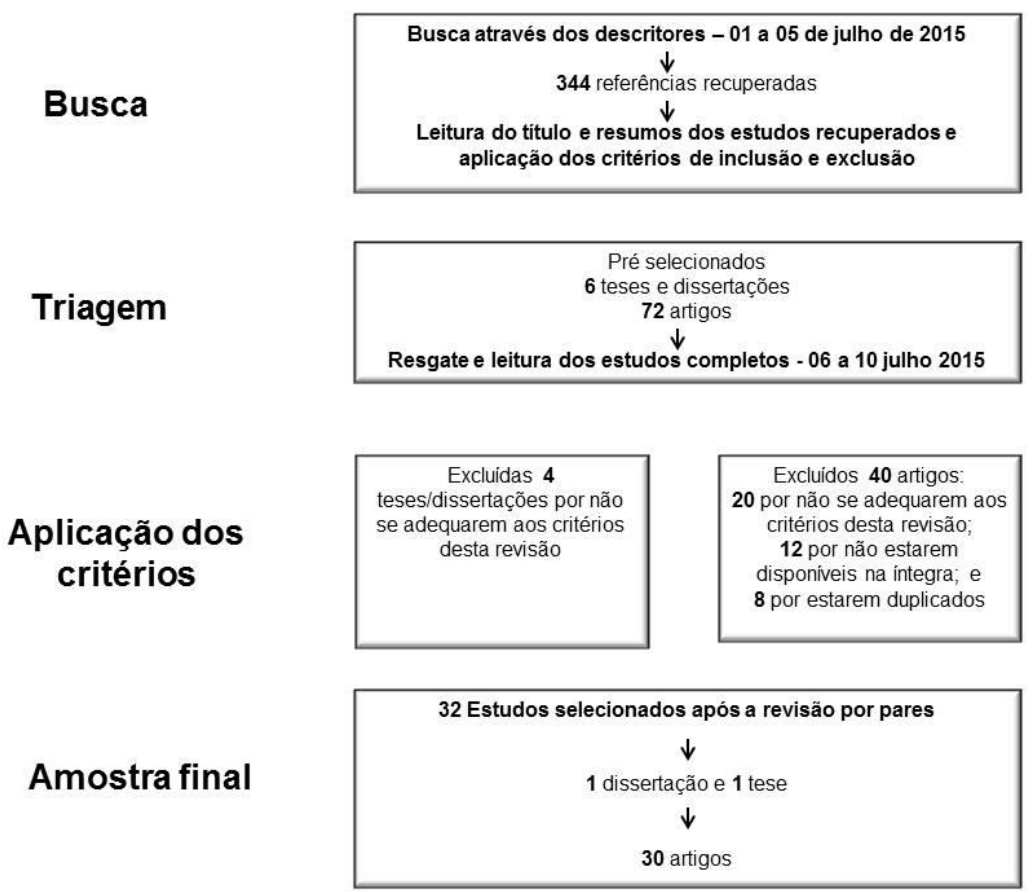

Figura 1 - Seleção da amostra. Florianópolis, SC, Brasil, 2015

\section{RESULTADOS}

\section{Características dos estudos encontrados}

Os estudos eleitos para esta revisão estão apresentados no Quadro 1. A seguir foram descriminados os achados em relação aos autores e títulos dos artigos.

Quadro 1 - Publicações acerca dos erros de medicação que envolve a equipe de enfermagem. Florianópolis, SC, Brasil, 2015 (continua)

\begin{tabular}{|l|l|}
\hline Autores & Título \\
\hline TEIXEIRA $^{(11)}$ & $\begin{array}{l}\text { Análise de causa raiz de incidentes relacionados à segurança do paciente na assistência } \\
\text { de enfermagem em unidades de internação, de um hospital privado, no interior do } \\
\text { Estado de São Paulo }\end{array}$ \\
\hline DALMOLIN ${ }^{(12)}$ & Erros de medicação no ambiente hospitalar: uma abordagem através da bioética complexa \\
\hline FONTANA, et al ${ }^{(13)}$ & $\begin{array}{l}\text { Análise documental da mídia escrita sobre eventos adversos ocorridos na prática da } \\
\text { enfermagem }\end{array}$ \\
\hline${\text { LOPES, et al }{ }^{(14)}}^{\text {ROQUE; } \text { MELO }^{(15)}}$ & Análise da rotulagem de medicamentos semelhantes: potenciais erros de medicação \\
\hline BOHOMOL & Avaliaça dos eventos adversos a medicamentos no contexto hospitalar \\
\hline $\begin{array}{l}\text { BELELA; PEDREIRA; } \\
\text { PETERLINI }{ }^{(17)}\end{array}$ & $\begin{array}{l}\text { Erros de medicação: estudo descritivo das classes dos medicamentos e medicamentos } \\
\text { de alta vigilância }\end{array}$ \\
\hline $\begin{array}{l}\text { CORBELLINI, et } \\
\text { al(18) }\end{array}$ & $\begin{array}{l}\text { Eventos de medicação em pediatria } \\
\text { enfermagem }\end{array}$ \\
\hline $\begin{array}{l}\text { VELOSO; TELLES } \\
\text { FILHO; DURÃO }\end{array}$ & $\begin{array}{l}\text { Identificação e análise de erros no preparo de medicamentos em uma unidade pediátrica } \\
\text { hospitalar }\end{array}$ \\
\hline $\begin{array}{l}\text { YAMAMOTO; } \\
\text { PETERLINI; } \\
\text { BOHOMOL }\end{array}$ & Notificação espontânea de erros de medicação em hospital universitário pediátrico \\
\hline
\end{tabular}




\begin{tabular}{|c|c|}
\hline PICHLER, et al (21) & $\begin{array}{l}\text { Erros de medicação: análise ergonômica de utensílios da sala de medicação em ambiente } \\
\text { hospitalar }\end{array}$ \\
\hline $\begin{array}{l}\text { PARANAGUÁ, et } \\
\mathrm{al}^{(22)}\end{array}$ & $\begin{array}{l}\text { Prevalência e fatores associados aos incidentes relacionados à medicação em pacientes } \\
\text { cirúrgicos }\end{array}$ \\
\hline CAMERINI; SILVA ${ }^{(23)}$ & $\begin{array}{l}\text { Segurança do paciente: análise do preparo de medicação intravenosa em hospital da } \\
\text { rede sentinela }\end{array}$ \\
\hline $\begin{array}{l}\text { LORENZINI; SANTI; } \\
\text { BÁO }^{(24)}\end{array}$ & $\begin{array}{l}\text { Segurança do paciente: análise dos incidentes notificados em um hospital do sul do } \\
\text { Brasil }\end{array}$ \\
\hline HARADA, et al ${ }^{(25)}$ & Segurança na administração de medicamentos em pediatria \\
\hline SILVA; CAMERINI ${ }^{(26)}$ & Análise da administração de medicamentos intravenosos em hospital da rede sentinela \\
\hline $\begin{array}{l}\text { TEIXEIRA; } \\
\text { CASSIANI }\end{array}$ & Análise de causa raiz de acidentes por quedas e erros de medicação em hospital \\
\hline $\begin{array}{l}\text { ROZENFEL; } \\
\text { GIORDANI; } \\
\text { COELHO }^{(28)}\end{array}$ & Eventos adversos a medicamentos em hospital terciário: estudo piloto com rastreadores \\
\hline SILVA; CASSIANI ${ }^{(29)}$ & $\begin{array}{l}\text { Análise prospectiva de risco do processo de administração de medicamentos anti- } \\
\text { infecciosos }\end{array}$ \\
\hline LOPES, et $\mathrm{al}^{(30)}$ & $\begin{array}{l}\text { Erros de medicação realizados pelo técnico de enfermagem na UTI: contextualização da } \\
\text { problemática }\end{array}$ \\
\hline SILVA, et $\mathrm{al}^{(31)}$ & Eventos adversos a medicamentos em um hospital sentinela do Estado de Goiás, Brasil \\
\hline $\begin{array}{l}\text { LEMOS; SILVA; } \\
\text { MARTINE(32) }^{\text {MART }}\end{array}$ & $\begin{array}{l}\text { Fatores que predispõem à distração da equipe de enfermagem durante o preparo e a } \\
\text { administração de medicamentos }\end{array}$ \\
\hline $\begin{array}{l}\text { PRAXEDES; TELLES } \\
\text { FILHO }^{(33)}\end{array}$ & $\begin{array}{l}\text { Erros e ações praticadas pela instituição hospitalar no preparo e administração de } \\
\text { medicamentos }\end{array}$ \\
\hline FERREIRA, et al ${ }^{(34)}$ & $\begin{array}{l}\text { Evento adverso versus erro de medicação: percepções da equipe de enfermagem atuante } \\
\text { em terapia intensiva }\end{array}$ \\
\hline $\begin{array}{l}\text { SHAHROKHI; } \\
\text { EBRAHIMPOUR; } \\
\text { GHODOUS }^{(35)}\end{array}$ & Factors effective on medication errors: a nursing view \\
\hline LAWTON, et al ${ }^{(36)}$ & $\begin{array}{l}\text { Identifying the latent failures underpinning medication administration errors: an } \\
\text { exploratory study }\end{array}$ \\
\hline MARQUET, et al ${ }^{(37)}$ & $\begin{array}{l}\text { One fourth of unplanned transfers to a higher level of care are associated with a highly } \\
\text { preventable adverse event: a patient record review in six Belgian hospitals }\end{array}$ \\
\hline VALENTIN, et al ${ }^{(38)}$ & $\begin{array}{l}\text { Safety climate reduces medication and dislodgement errors in routine intensive care } \\
\text { practice }\end{array}$ \\
\hline CHANG; MARK ${ }^{(39)}$ & Moderating effects of learning climate on the impact of RN staffing on medication errors \\
\hline PAQUET, et al ${ }^{(40)}$ & $\begin{array}{l}\text { Psychosocial work environment and prediction of quality of care indicators in one } \\
\text { Canadian health center }\end{array}$ \\
\hline $\begin{array}{l}\text { WEST; PATRICIAN; } \\
\text { LOAN }^{(41)}\end{array}$ & Staffing matters - every shift \\
\hline MATTOX $^{(42)}$ & for improving patient safety: linking task type to error type \\
\hline
\end{tabular}

Em relação ao idioma, predominou o português em 24 estudos $(72 \%)$ e as publicações em inglês somaram oito (28\%). Quanto ao país de publicação, o destaque foi para o Brasil com 22 estudos, Estados Unidos com nove e Nova Zelândia com um estudo. Houve predomínio também, de 21 estudos de natureza quantitativa ( $66 \%$ ), em seguida os qualitativos somaram sete $(22 \%)$ e os ensaios teóricos ocuparam $12 \%$ dos achados, com quatro estudos.

Para a coleta de dados predominou a pesquisa documental em 12 publicações (37\%), utilizando como fonte prontuários de pacientes, notificações de eventos adversos e notícias veiculadas na mídia jornalística; entrevistas e questionários aparecem em 10 (32\%); observação não participante foi utilizada em oito (28\%); dois estudos fizeram uso da análise fotográfica e um de estudo de caso. 


\section{Relação da enfermagem com os erros de medicação}

A relação da equipe de enfermagem como os erros praticados com a medicação foi dividida em duas subcategorias: relação direta - referente às causas dos erros relacionadas diretamente com a equipe de enfermagem; e relação indireta - no tocante às causas associadas a outros membros da equipe de saúde ou a algum outro momento do processo de administração de medicamentos.

\section{Relação direta}

As causas relacionadas diretamente à enfermagem estão dispostas na Figura 2.

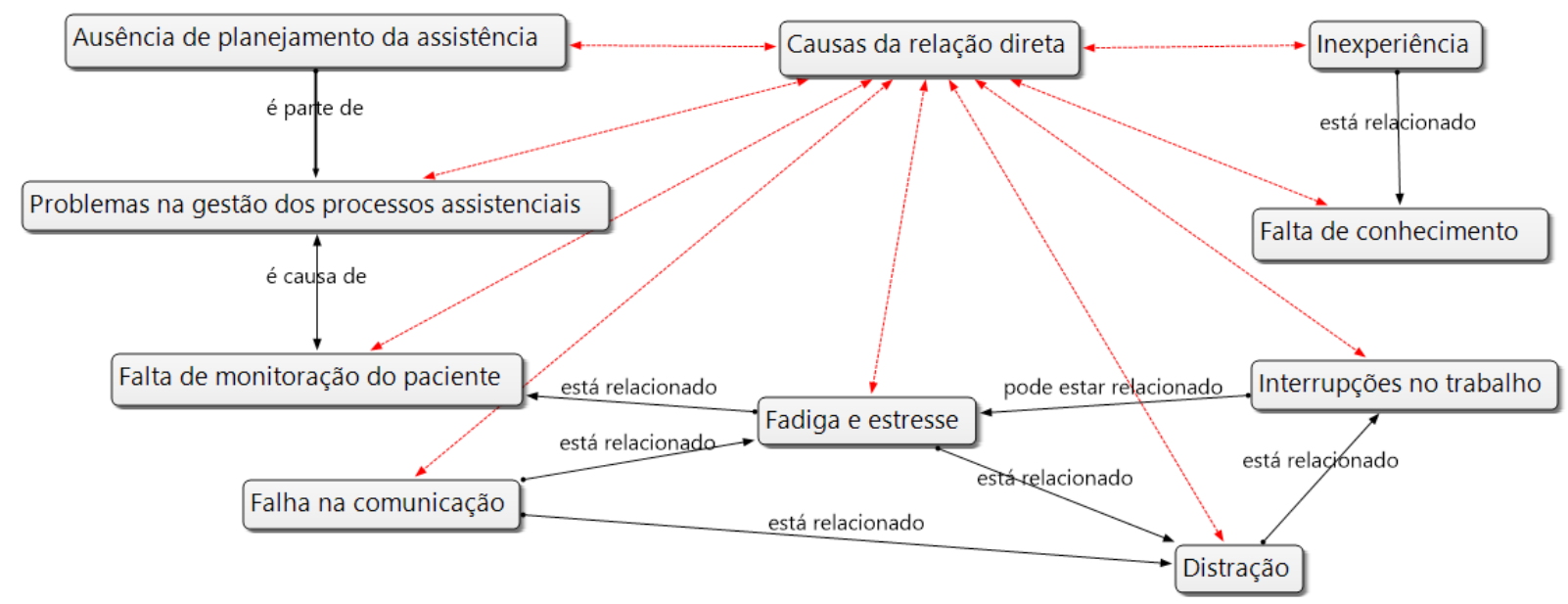

Figura 2 - Causas da relação direta. Florianópolis, SC, Brasil, 2015

As causas que podem ser atribuídas diretamente à equipe de enfermagem aparecem claramente nos estudos encontrados. A grande maioria deles se referia aos erros no preparo e na administração de medicamentos (21 estudos).

Destaque importante para a questão da fadiga e do estresse que está intimamente relacionada com o processo de trabalho de enfermagem configurado de forma desgastante na maioria das instituições de saúde.

\section{Relação indireta}

A relação indireta que a equipe de enfermagem enfrenta diante dos erros com a medicação provém de 06 fatores diferentes, entretanto, altamente relacionados entre si conforme Figura 3.

As causas indiretas dos erros de medicação estão associadas aos problemas de gestão das instituições, no que concernem as condições de trabalho, a problemas oriundos da ação de outros profissionais - médicos e farmacêuticos, problemas com a produção de medicamentos e, com as condições do próprio paciente.

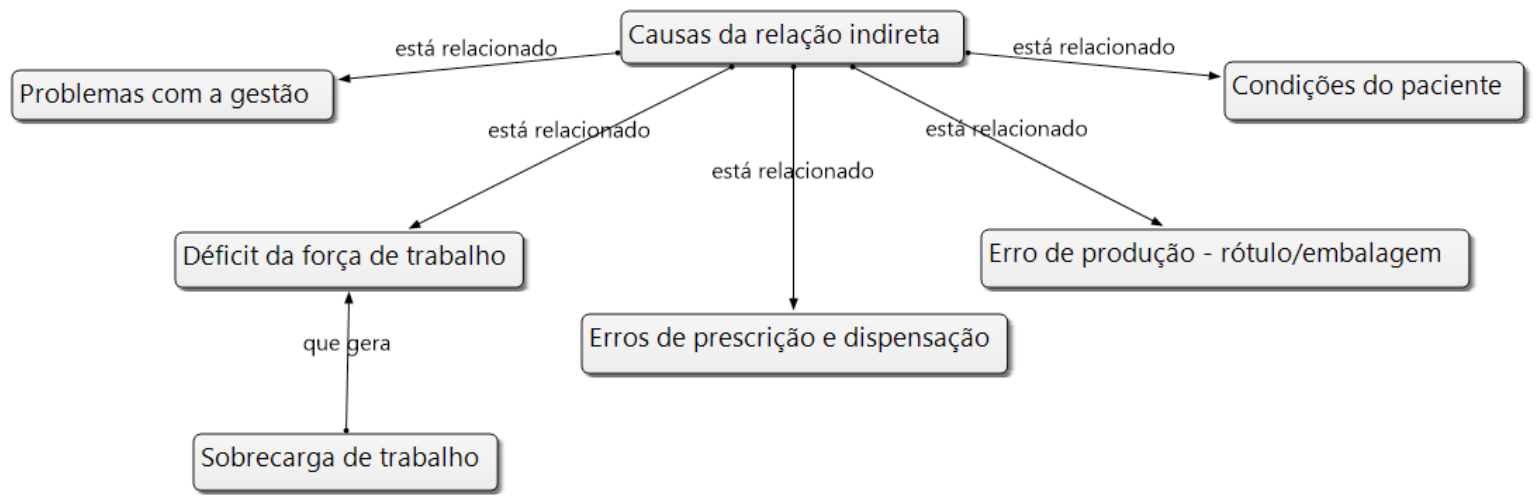

Figura 3 - Causas da relação indireta. Florianópolis, SC, Brasil, 2015 
Os erros com maior destaque na literatura estão relacionados diretamente com a equipe de enfermagem, pois sua origem está justamente no final do processo de administração de medicamentos, são eles os erros de horário e de velocidade de infusão ${ }^{(11,12,18,20,23,26,29-32,38)}$. Além do fato de terem ênfase nos estudos, esses erros chamam a atenção pelo desfecho que se inicia na falha da terapêutica até o óbito do paciente.

A relação direta sofre a influência da fadiga e do estresse gerados no trabalho. O estresse e o cansaço são considerados causas frequentes de erros de medicação, como os erros de dose, de horário, de técnicas e de velocidade de gotejamento ${ }^{(43)}$. O estresse influencia negativamente os relacionamentos entre os profissionais e torna a assistência mecanizada, o que potencializa as condições para que o erro ocorra ${ }^{(44)}$.

A falta de planejamento e também a falta de experiência e de conhecimento da equipe de enfermagem estiveram diretamente ligadas à produção do erro. Déficits de conhecimento e de experiência foram identificados em situações em que estavam atuando estudantes de enfermagem de nível técnico e graduação, sem supervisão (11 estudos). O conhecimento inadequado da medicação, no que tange à terapêutica das drogas, formas alternativas e cálculos de dosagens inadequadas são responsáveis por muitos dos episódios envolvendo a medicação ${ }^{(45,46)}$.

As falhas de comunicação e as distrações aparecem como fatores predisponentes ao erro, sendo que a comunicação eficiente minimiza em muito o erro, a ponto até de impedir que ele aconteça ${ }^{(4,47)}$. A distração provém de inúmeros fatores presentes no cotidiano dos profissionais, como telefone celular, televisão, conversas entre a equipe e outros profissionais, dentre outros.

Da relação indireta, há que se destacar as condições do paciente no momento da assistência. Esta revisão constatou que a maior frequência de erros ocorre nas Unidades de Internação (nove estudos), nas Unidades de Terapia Intensiva (UTI) (cinco estudos) e na Pediatria (quatro estudos), o que pode ser explicado pelas características dos usuários dessas unidades, que são pacientes mais vulneráveis fisicamente, com grande quantidade de medicações e procedimentos e pela alta demanda de trabalho exigida da equipe de enfermagem. Consequentemente, esse fator relacionado ao paciente, influenciará a fadiga e o estresse. Quanto mais grave o paciente, mais cuidados são necessários, e nem sempre as instituições de saúde dispõem de profissionais de enfermagem em número suficiente, o que contribui para a sobrecarga de trabalho e estresse físico e mental dos trabalhadores.

Os erros de prescrição e dispensação aparecem em seis estudos e ressaltam que esses erros configuram falha nas barreiras que antecedem a administração de medicamentos pela enfermagem. Em estudo conduzido em países do Oriente Médio as taxas de erros de dispensação e prescrição variaram de $7,1 \%$ a $90,5 \%$, sendo que os erros mais comuns foram a dose e a frequência erradas ${ }^{(48)}$.

Ao analisar a influência da redação da prescrição médica nos erros de via de administração, em estudo realizado ${ }^{(46)}$, os autores constataram que $91,3 \%$ das prescrições continham siglas/abreviaturas, $22,8 \%$ não continham dados do paciente e 4,3\% não apresentavam data e continham rasuras. Esses erros são comuns e podem desencadear uma série de problemas no processo de administração de medicamentos. Os autores atestam ainda, que outros fatores contribuem também para os erros de prescrição, como a falta de conhecimento, nome da droga, cálculos de dosagens e formulações inapropriadas $^{(46)}$.

Evidências apontam que os profissionais de saúde desenvolvem um trabalho no qual pode ocorrer erros de medicação, entretanto, as condições de trabalho oferecidas pelas instituições de saúde têm forte influência na geração do erro ${ }^{(4)}$. O déficit na força de trabalho, que gera a sobrecarga de trabalho tem significativa ligação com os erros de medicação, problemática essa que precisa ser considerada pelos gestores dos serviços de saúde e pelos formuladores de políticas ${ }^{(4,49,50)}$. Por isso, pensar a segurança do paciente requer necessariamente repensar as condições de trabalho da enfermagem.

A problemática do erro de medicação se estende para fora dos muros das instituições de saúde, devido aos erros de produção dos medicamentos. O estudo que teve por objetivo a rotulagem de medicamentos, os resultados são preocupantes, pois $43 \%$ dos medicamentos eram possivelmente 
semelhantes e $44 \%$ tinham a mesma cor do rótulo ou da embalagem, configurando um forte potencial para erros de dispensação, armazenamento e administração de medicamentos ${ }^{(14)}$.

Os erros associados com a rotulagem das medicações ocorrem com frequência nas diversas formas de apresentação. Para mudar essa realidade é imprescindível enfrentá-la de diversas maneiras, com diferentes focos: tecnologia, educação e cultura de segurança são exemplos de estratégias para reduzir, potencialmente, os erros de medicação ${ }^{(51)}$.

\section{CONSIDERAÇÕES FINAIS}

Constatou-se por meio desta revisão que a enfermagem tem forte ligação com os erros de medicação. Essa ligação é explicada, especialmente, por dois tipos de relação: a direta e a indireta. A relação direta é a que mais traz implicações para o processo de trabalho da enfermagem, pois reproduz as adversidades da própria profissão, como a formação e conhecimento deficitários, o estresse vivenciado diariamente pelas duplas ou triplas jornadas de trabalho, as falhas de comunicação entre os componentes da equipe e a distração.

A relação indireta pode ser explicada pelo fato de que a enfermagem, muito embora não tenha sido a responsável primária pelo erro no processo de medicação, é considerada como a última barreira para evitar que o erro chegue ao paciente. Nesse contexto, todos os erros que antecedem a administração de medicamentos podem ser evitados ou minimizados através das ações da enfermagem.

Conclui-se então que os mecanismos de prevenção dos erros têm que obrigatoriamente passar por todos os profissionais envolvidos no sistema de medicação, para que todos sejam igualmente responsáveis pelo desfecho. A assistência de saúde é, majoritariamente, um trabalho coletivo no qual as responsabilidades também devem ser compartilhadas. Este estudo possui limitações, que podem ter reduzido a amostra, referentes à escolha dos descritores e dos idiomas e a ausência do texto completo de alguns estudos, tendo em vista a forma de acesso gratuito via Universidade Federal de Santa Catarina.

\section{REFERÊNCIAS}

1. Ministério da Saúde (BR). Agência Nacional de Vigilância Sanitária. Assistência segura: uma reflexão teórica aplicada à prática. Série Segurança do Paciente e Qualidade em Serviços de Saúde. Anvisa; 2013.

2. Wulff K, Cummings GG, Marck P, Yurtseven O. Medication administration technologies and patient safety: a mixed-method systematic review. J. adv. nurs. [Internet] 2011; 67(10) [acesso em 13 jul 2015]. Disponível: https://dx.doi.org/ 10.1111/j.1365-2648.2011.05676.x

3. National Coordinating Council for Medication Error Reporting and Prevention (NCCMERP). United States: National Coordinating Council. [Internet] 1998-2010 [acesso em 14 jun 2014]. Disponível:

http://www.nccmerp.org/about-medication-errors

4. Keers RN, Williams SD, Cooke J, AshcroftDM. Causes of medication administration errors in hospitals: a systematic review of quantitative and qualitative evidence. Drug saf. [Internet] 2013; 36(11) [acesso em 13 jul 2015]. Disponível: https://dx.doi.org/10.1007/s40264-013-0090-2

5. Aranaz-Andrés JMR, Aibar-Remón C, Limón-Ramírez R, Amarilla A, Restrepo FR, Urroz O, et al. Prevalence of adverse events in the hospitals of five Latin American countries: results of the "Iberoamerican study of adverse events" (IBEAS). BMJ Qual Saf. [Internet] 2011; 20(12) [acesso em 13 jul 2015]. Disponível:

https://dx.doi.org/10.1136/bmjqs.2011.051284

6. Mansour M, James V, Edgley A. Investigating the safety of medication administration in adult critical care settings. Nurs. crit. care. [Internet] 2012; 17(4): 189-97. [acesso em 13 jul 2015]. Disponível: doi: 10.1111/j.14785153.2012.00500.x

7. Fassarella CS, Bueno AAB, Souza ECC. Segurança do paciente no ambiente hospitalar: os avanços na prevenção de eventos adversos no sistema de medicação. Rev. rede cuid. saúde. [Internet] 2013; 7(1): 1-8. [acesso em 13 jul 
8. Whittemore R, Knafl K. The integrative review: updated methodology. J. adv.nurs. [Internet] 2005; 52(5): 546-53. [acesso em 13 jul 2015]. Disponível: doi/10.1111/j.1365-2648.2005.03621.x/epdf

9. Polit D, Beck C. Nursing research: generating and assessing evidence for nursing practice. Hong Kong: Lippincott, Williams \& Wilkins; 2012. Literature reviews: finding and critiquing evidence. 94-125.

10. Bardin L. Análise de conteúdo. 1. ed. rev. e ampl. Lisboa: Edições 70; 2011.

11. Teixeira TCA. Análise de causa raiz de incidentes relacionados à segurança do paciente na assistência de enfermagem em unidades de internação, de um hospital privado, no interior do Estado de São Paulo [tese]. Ribeirão Preto (SP): Universidade de São Paulo; 2012.

12. Dalmolin GRS. Erros de medicação no ambiente hospitalar: uma abordagem através da bioética complexa [dissertação]. Porto Alegre: Universidade Federal do Rio Grande do Sul; 2012.

13. Fontana RT, Wolf J, Rodrigues FCP, Castro LM. Análise documental da mídia escrita sobre eventos adversos ocorridos na prática da enfermagem. Rev enferm UFPE on line. [Internet] 2015; 9(4): 8103-10. [acesso em 13 jul 2015]. Disponível: DOI: 10.5205/reuol.6235-53495-1-RV.0904supl201516

14. Lopes DMA, Néri EDR, Madeira LS, Souza Neto PJ, Lélis ARA, de Souza TR, et al. Análise da rotulagem de medicamentos semelhantes: potenciais erros de medicação. Rev. Assoc. Med. Bras. [Internet] 2012; 58(1): 95-103. [acesso em 02 ago 2015]. Disponível: http://www.scielo.br/pdf/ramb/v58n1/v58n1a21.pdf

15. Roque KE, Melo ECP. Avaliação dos eventos adversos a medicamento. Esc Anna Nery rev enferm. [Internet] 2012; 16(1): 121-7. [acesso em 02 ago 2015]. Disponível: http://www.scielo.br/pdf/ean/v16n1/v16n1a16.pdf

16. Bohomol E. Erros de medicação: estudo descritivo das classes dos medicamentos e medicamentos de alta vigilância. Esc Anna Nery Rev enferm. [Internet] 2014; 18(2): 311-6. [acesso em 02 ago 2015]. Disponível:

http://www.scielo.br/pdf/ean/v18n2/en_1414-8145-ean-18-02-0311.pdf

17. Belela ASC, Pedreira MLG, Peterlini MAS. Erros de medicação em pediatria. Rev bras enferm. [Internet] 2011; 64(3): 563-9. [acesso em 02 ago 2015]. Disponível:

http://www.scielo.br/scielo.php?script=sci_arttext\&pid=S0034-71672011000300022

18. Corbellini VL, Schilling MC, Frantz SF, Godinho TG, Urbanetto JS. Eventos adversos relacionados a medicamentos: percepção de técnicos auxiliares de enfermagem. Rev bras enferm. [Internet] 2011; 64(2): 241-7. [acesso em 02 ago 2015]. Disponível: http://www.redalyc.org/articulo.oa?id=267019461004

19. Veloso IR, Telles Filho PCP, Durão AMS. Identificação e análise de erros no preparo de medicamentos em uma unidade pediátrica hospitalar. Rev gaúcha [Internet] enferm. 2011; 2(1): 93-9. [acesso em 02 ago 2015]. Disponível: http://www.scielo.br/pdf/rgenf/v32n1/a12v32n1.pdf

20. Yamamoto, M. S., Peterlini, M. A. S., \& Bohomol, E. (2015). Notificação espontânea de erros de medicação em hospital universitário pediátrico. Acta Paulista de Enfermagem, 24(6), 766-771.

21. Pichler RF, Garcia LJ, Seitz EM, Merino GSAD, Gontijo LA, Merin EAD. Erros de medicação: análise ergonômica de utensílios da sala de medicação em ambiente hospitalar. Cad saúde coletiva. 2014; 22(4): 365-71.

22. Paranaguá TTB, Bezerra ALQ, Santos ALM, Silva AEBC. Prevalência e fatores associados aos incidentes relacionados à medicação em pacientes cirúrgicos. Rev esc enferm USP. 2014; 48(1): 41-8.

23. Camerini FG, Silva LD. Segurança do paciente: análise do preparo de medicação intravenosa em hospital da rede sentinela. Texto \& Contexto enferm. [Internet] 2011; 20(1): 41-9. [acesso em 02 ago 2015]. Disponível: http://www.scielo.br/pdf/tce/v20n1/05.pdf

24. Lorenzini E, Santi JAR, Báo ACP. Segurança do paciente: análise dos incidentes notificados em um hospital do sul do Brasil. Rev gaúcha enferm. 2014; 35(2):121-7.

25. Harada MJCS, Chanes DC, Kusahara DM, Pedreira MLG. Segurança na administração de medicamentos em Pediatria. Acta paul enferm. 2012; 25(4): 639-42. 
26. Silva LD, Camerini FG. Análise da administração de medicamentos intravenosos em hospital da rede sentinela. Texto \& Contexto enferm. [Internet] 2012; 21(3): 633-41. [acesso em 02 ago 2015]. Disponível:

http://www.scielo.br/scielo.php?script=sci_arttext\&pid=S0104-07072012000300019

27. Teixeira TCA, Cassiani SHB. Análise de causa raiz de acidentes por quedas e erros de medicação em hospital. Acta paul enferm. 2014; 27(2): 100-7.

28. Rozenfeld S, Giordani F, Coelho S. Eventos adversos a medicamentos em hospital terciário: estudo piloto com rastreadores. Rev. saúde pública. 2013; 47(6): 1102-11.

29. Silva AEBC, Cassiani SHB. Análise prospectiva de risco do processo de administração de medicamentos antiinfecciosos. Rev latinoam enferm. [Internet] 2013; 21(Spec). [acesso em 02 ago 2015]. Disponível: http://www.scielo.br/scielo.php?pid=S0104-11692013000700029\&script=sci_arttext\&tlng=pt

30. Lopes BC, Vargas MAO, Azeredo NSG, Behenck A. Erros de medicação realizados pelo técnico de enfermagem na UTI: contextualização da problemática. Enferm foco. 2013; 3(1): 16-21.

31. Silva AEBC, Reis AMM, Miasso AI, Santos JO, Cassiani SHB. Eventos adversos a medicamentos em um hospital sentinela do Estado de Goiás, Brasil. Revista latinoam enferm. [Internet] 2011; 19(2): 09 telas. [acesso em 02 ago 2015]. Disponível: http://www.scielo.br/pdf/rlae/v19n2/pt_21

32. Lemos NRF, Silva VR, Martinez MR. Fatores que predispõem à distração da equipe de enfermagem durante o preparo e a administração de medicamentos. REME Rev min enferm. 2012; 16(2): 201-7.

33. Praxedes MFS, Telles Filho PCP. Erros e ações praticadas pela instituição hospitalar no preparo e administração de medicamentos. REME Rev min enferm. 2011; 15(3): 406-11.

34. Ferreira PC, Dantas ALM, Diniz KD, Ribeiro KRB, Machado RC, Tourinho FSV. Evento adverso versus erro de medicação: percepções da equipe de enfermagem atuante em terapia intensiva. Rev pesqui cuid findam. 2014; 2: 725-34.

35. Shahrokhi A, Ebrahimpour F, Ghodousi A. Factors effective on medication errors: a nursing view. J res pharm pract. 2013; 2(1): 18-23.

36. Lawton R, Carruthers S, Gardner P, Wright J, McEachan RR. Identifying the latent failures underpinning medication administration errors: an exploratory study. Health serv res. [Internet] 2012; 47(4): 1437-59. [acesso em 02 ago 2015]. Disponível: http://www.ncbi.nlm.nih.gov/pmc/articles/PMC3401393/

37. Marquet K, Claes N, De Troy E, Kox G, Droogmans M, Schrooten W, et al One fourth of unplanned transfers to a higher level of care are associated with a highly preventable adverse event: a patient record review in six belgian hospitals. Crit care med. 2015; 43(5): 1053-61.

38. Valentin A, Schiffinger M, Steyrer J, Huber C, Strunk G. Safety climate reduces medication and dislodgement errors in routine intensive care practice. Intensive care med. 2013; 39: 391-8.

39. Chang Y, Mark B. Moderating effects of learning climate on the impact of RN staffing on medication errors. Nurs res. 2011; 60(1): 32-9.

40. Paquet M, Courcy F, Lavoie-Tremblay M, Gagnon S, Maillet S. Psychosocial work environment and prediction of quality of care indicators in one canadian health center. Worldviews Evid Based Nurs. [Internet] 2013 May; 10(2): 82-94. Disponível: doi: 10.1111/j.1741-6787.2012.00250.x.

41. West G, Patrician APA, Loan L. Staffing matters - every shift. Am j nurs. [Internet] 2012; 112(12): 22-7. [acesso em 06 ago 2015]. Disponível: 10.1097/01.NAJ.0000423501.15523.51

42. Mattox EA. Strategies for improving patient safety: linking task type to error type. Crit care nurs. [Internet] 2012; 32(1): 52-78. [acesso em 10 ago 2015]. Disponível: doi: 10.4037/ccn2012303.

43. Santana JCB, de Sousa MA, Soares HC, Avelino KSA. Fatores que influenciam e minimizam os erros na administração de medicamentos pela equipe de enfermagem Rev. Enferm. Revista. [Internet] 2012; 15(1) [acesso em 13 ago 2015]. Disponível: http://periodicos.pucminas.br/index.php/enfermagemrevista/article/view/3300/3657

44. Martins CCF, Santos VEP, Pereira MS, Santos NP. Relacionamento interpessoal da equipe de enfermagem $\mathrm{x}$ 
estresse: limitações para a prática. Cogitare enferm. [Internet] 2014; 19(2) [acesso 02 set 2015]. Disponível: http://dx.doi.org/10.5380/ce.v19i2.36985

45. Mansouri A, Ahmadvand A, Hadjibabaie M, Javadi M, Khoee SH, Dastan F, et al. A review of medication errors in iran: sources, under report in greasons and preventive measures. Iran J.Pharm. Res. [Internet] 2014; 13(1): 3-17. [acesso 02 set 2015]. Disponível: http://ijpr.sbmu.ac.ir/article_1443_68.html

46. Gimenes FRE, Marques TC, Teixeira TCA, Mota MLS, Silva AEBC, Cassiani SHB. Administração de medicamentos, em vias diferentes das prescritas, relacionada à prescrição médica. Rev. latino-am. enferm. [Internet] 2011; 19(1) [acesso em 02 ago 2015]. Disponível: http://dx.doi.org/10.1590/S0104-11692011000100003

47. Smeulers M,Verweij L, Maaskant JM, de Boer M, Krediet CT, Nieveen van Dijkum EJ, et al. Quality indicators for safe medication preparation and administration: a systematic review. PLos ONE [Internet] 2015; 10(4) [acesso em 02 ago 2015]. Disponível: https://dx.doi.org/10.1371/journal.pone.0122695

48. Alsulami Z, Conroy S, Choonara I. Medication errors in the Middle East countries: a systematic review of the literature. Eur J Clin Pharmacol. [Internet] 2013; 69(4) [acesso em 02 ago 2015]. Disponível:

https://dx.doi.org/10.1007/s00228-012-1435-y

49. Treiber LA, Jones JH. Medication errors, routines, and differences between perioperative and nonperioperative nurses. AORN j. [Internet] 2012; 96(3): 285-94. [acesso em 02 ago 2015]. Disponível: doi: 10.1016/j. aorn.2012.06.013.

50. Vazin A, Delfani S. Medication errors in an internal intensive care unit of a large teaching hospital: a direct observation study. Acta med. iran. [Internet] 2012; 50(6): 425-32 [acesso em 02 ago 2015]. Disponível:

http://acta.tums.ac.ir/index.php/acta/article/view/3924

51. Kruer RM, Jarrell AS, Latif A. Reducing medication errors in critical care: a multimodal approach. Clin Pharmacol. [Internet] 2014; 6: 117-26. [acesso em 02 ago 2015]. Disponível: http://www.ncbi.nlm.nih.gov/pmc/ articles/PMC4155993/ 